\title{
Investigation of the Effects of Chirped RZ Signals in Reducing the Transmission Impairments in R-SOA-Based Bidirectional PONs
}

\author{
Luca Banchi, Raffaele Corsini, Marco Presi, and Ernesto Ciaramella, Member, IEEE
}

\begin{abstract}
Distributed and concentrated reflections represent the two main limitations in reflective-semiconductor optical amplifier (R-SOA)-based passive optical networks (PONs). In this paper, we experimentally discuss how the use of chirped signals in centralized light seeding bidirectional PON can increase the resilience of the system against those two types of reflections. An experimental comparison of the performance of a highly chirped return to zero (RZ) modulation format and the nonreturn to zero is given. Error-free operation is achieved down to $10 \mathrm{~dB}$ of signal to crosstalk ratio in presence of distributed reflection, when the upstream signal is highly chirped RZ signal. The same chirped modulation leads to a tolerance of more than $-25 \mathrm{~dB}$ network return loss due to concentrated reflections. Finally, we assess also the system feasibility of a R-SOA-based full-duplex PON where both the upstream and the downstream are modulated signals.
\end{abstract}

Index Terms-Access networks, concentrated reflections, distributed reflections, nonreturn to zero (NRZ), optical crosstalk, passive optical network (PON), reflective semiconductor optical amplifier (R-SOA), return to zero (RZ).

\section{INTRODUCTION}

$\mathbf{T}$ HE rapid development of the passive optical networks (PONs) is driven by the increased request for high capacity and flexibility in the access network. Wavelength division multiplexing-PONs (WDM-PONs) are a promising solution for low-cost and simple design fiber-to-the-home connectivity [1 ]-[3]. Among them, WDM-PONs based on reflective structures at the optical network unit (ONU), recently attracted a lot of interests mainly thanks to their cost effectiveness and flexibility [2]. Particularly, wavelength independent reflective remodulators, such as reflective semiconductor optical amplifiers (R-SOAs), open the way to the deployment of single-feeder loopback PON with centralized light seeding (CLS) [4 ]. In these networks, a continuous light is distributed over a fiber link form the optical line terminal (OLT) to each ONU. At the ONU side, the continuous wave (CW) light is then modulated and transmitted back over the same fiber. Although this approach allows for a single fiber loop-back network architecture, it suffers from two well-known fundamental limitations: the reflections distributed along the fiber [5 ]-[9], and

Manuscript received September 15, 2010; revised January 24, 2011; accepted February 20, 2011. Date of publication February 28, 2011; date of current version April 01, 2011. This work was supported in part by Ericsson under a Grant.

The authors are with Scuola Superiore Sant'Anna, Pisa 56124, Italy (e-mail: 1.banchi@sssup.it; r.corsini@sssup.it; marco.presi@sssup.it; e.ciaramella@sssup.it).

Color versions of one or more of the figures in this paper are available online at http://ieeexplore.ieee.org.

Digital Object Identifier 10.1109/JLT.2011.2120596 the concentrated reflections in the optical distribution network [7]. The distributed reflections arise along the fiber because of Rayleigh back-scattering (RBS) of the optical signal. In bidirectional PONs, the effect of back-scattered light can be classified into two types: reflections type-I and type-II [9]. Type-I reflections occur because of the RBS of the seeding light in the fiber. Those type-I reflections are directed toward the OLT where they interfere with the upstream signal. Similarly, type-II reflections are due to the RBS of the upstream signal in the fiber, but they are directed toward the ONU where they mix with the upstream signal in the reflective modulator. Both types of reflection can coherently mix with the signal generating crosstalk interference, which severely deteriorate the system performance [10], [11]. The concentrated reflections are originated because of unpredictable reasons such as bad splicing, neighbor devices with low return loss or broken fibers. Those kind of reflections are particularly detrimental in the R-SOA-based PON, especially when they are generated close to the ONU because the loss between the reflection point and the ONU is very small [8]. Hence, they go back into the R-SOA with high power levels, they are amplified there and eventually copropagate with the upstream signal [12]. Both concentrated and distributed reflections induce a significant in-band interferometric crosstalk, which severely degrades the system performance, especially when the reflected light coherently mixes with the signal (coherent crosstalk) [13], [14].

Several techniques have been proposed to mitigate the effect of the in-band crosstalk in CLS PONs: in [15], a DC block was proposed to reduce the low-frequency beat noise generated during photodetection and in [16] the combined use of ad hoc line coding and postdetection filtering was exploited. As an alternative, it has been demonstrated that the in-band coherent crosstalk is effectively reduced by broadening the optical spectrum of the transmitted signals [5], [12], [17]. In order to broaden the upstream signal optical spectrum, in [12] a bias dithering is applied to the R-SOA. In [18], [19], the in-band crosstalk is reduced by the chirp induced combining a RF tone with the electrical data in the R-SOA driving signal. Those techniques move the crosstalk tolerance level to around $18 \mathrm{~dB}$. All these techniques are studied in a CLS-PON where the nonreturn-to-zero (NRZ) format is adopted for the upstream transmission. In [20], we proposed a novel technique based on the spectral broadening of the R-SOA-modulated output realized by using the return-to-zero (RZ) modulation format. We demonstrated that RZ modulation of the R-SOA broadens the optical spectrum of the upstream signal, which results in an increased 
tolerance to the distributed reflections. Using the RZ modulation of the R-SOA, we achieve the spectral broadening of the upstream signal with no need of bias dithering or external frequency modulation applied to the R-SOA.

In this paper, we present a comparative analysis of the performance of the RZ and the NRZ modulation format in R-SOA-based bidirectional CLS-PON in presence of distributed and concentrated reflections. We found that the transmission of highly chirped RZ signals in a bidirectional PON allows to reduce the coherent mixing between the signal and the reflected light, hence increases the resilience to the in-band coherent crosstalk. These results extend the technique proposed in [20], demonstrating how the RZ modulation of the upstream signal, can also successfully increase the resilience against the concentrated reflections. We compare the resilience to the in-band interferometric crosstalk obtained for the RZ and for the NRZ modulation format at different wavelengths. We demonstrate the proposed scheme which may be a valid alternative to other similar schemes, already known [15]-[19]. On the other hand, we strongly believe that it would be particularly interesting, as it could be straightforwardly implemented, whenever the PON is chosen to use RZ signals, e.g., in [21].

The paper is organized as follows. In Section II, we present the operating principle of the proposed solution. In Section III, the experimental setup is described. In Section IV, we compare the experimental results obtained for the RZ and the NRZ modulation formats in presence of distributed and concentrated reflections and we study the feasibility of a full-duplex PON based on the scheme proposed in [21].

\section{OPERATING PRINCIPLE}

It was demonstrated in [22] that the increased linewidth in a semiconductor results from the coupling between intensity and phase caused by the dependence of the refractive index on the carrier density in the semiconductor.

In the R-SOA, because of this gain-phase coupling, the modulated signal is accompanied by a phase modulation (chirp) given by [23]:

$$
\phi(t)=\frac{-\alpha_{\text {eff }}}{2} \ln (\Delta G(t))
$$

where $\alpha_{\text {eff }}$ is the effective linewidth enhancement factor measured at the output of the R-SOA and $\Delta G$ is the gain variation ( $\Delta G(t)=G(t)-G_{0}$, where $G_{0}$ is the average gain). Therefore, when $G(t)$ changes, the phase shift also changes, thus inducing frequency chirp. This phase modulation depends on the gain variations induced by the intensity transitions in the R-SOA driving signal, hence, in order to maximize the chirp on the modulated signal, we drive the R-SOA with a RZ format instead of the NRZ. When the R-SOA is driven by a NRZ signal, phase transitions occur only at 0-1 (or 1-0) transitions, whereas a constant symbol sequence (e.g., consecutive 1s) comes with no phase change. On the other hand, in the RZ signal, we have two opposite intensity transitions at every mark: therefore, the R-SOA-modulated output is always highly chirped. This difference of the chirp features in the NRZ and RZ format is illustrated in Fig. 1, where we sketch the phase changes (dashed-line) induced by the amplitude modulation (solid-line) for the two dif-

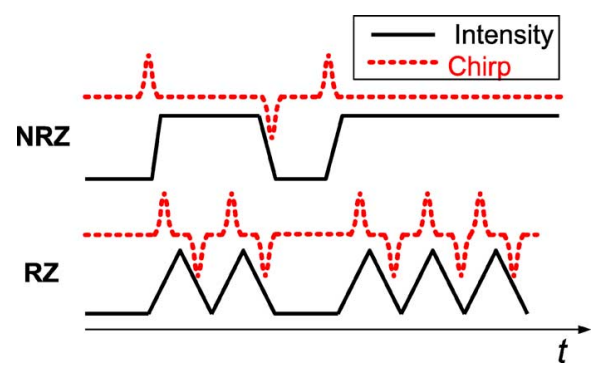

Fig. 1. Frequency chirp in the NRZ and RZ formats.

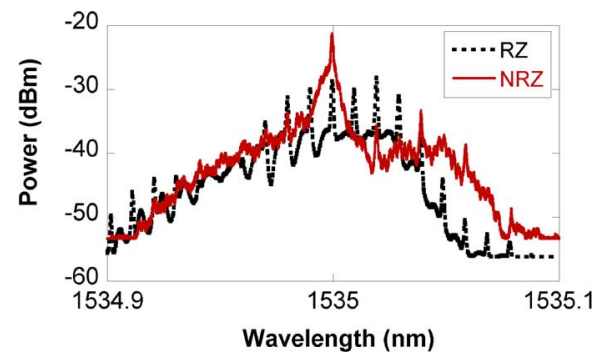

Fig. 2. Optical spectra of the RZ (dashed-line) and the NRZ (solid-line) modulated uplink signals at $1.25 \mathrm{~Gb} / \mathrm{s}$ (taken with a $20 \mathrm{MHz}$ resolution bandwidth OSA).

ferent modulation formats. As shown in the figure, consecutive symbols do not generate any phase modulation in the NRZ stream, whereas the RZ stream shows always frequency variations, which effectively broadens the transmitted signal optical spectrum and increases the resilience to the crosstalk. This spectral broadening is illustrated in Fig. 2, where we report the optical spectra of the RZ (dashed-line) and the NRZ (solid-line) outputs, with the same optical power $(6 \mathrm{dBm})$, taken with a 20 $\mathrm{MHz}$ resolution bandwidth optical spectrum analyzer (OSA). In the RZ spectrum, we observe the typical peaks equally spaced at the bit time (1.25 Gbits/s). Moreover, the chirp induced by the RZ modulation largely broadens the spectrum of the signal and makes it asymmetric and much wider than the spectrum obtained for NRZ modulation. This spectral broadening, due to the chirp, corresponds to a significant reduction of the coherence time of the modulated signal [24]. If the coherence time of the signal becomes smaller, also the impact of the coherent crosstalk is reduced, hence an improvement in the resilience against both type of reflections can be achieved [12].

\section{EXPERIMENTAL SETUP}

In order to evaluate the impact of both the distributed and the concentrated reflections on the proposed modulation format, we use the experimental setup reported in Fig. 3. A tunable laser (TL) emits the seeding light. The TL output light can be externally modulated or $\mathrm{CW}$. We first use the $\mathrm{CW}$ operation in order to characterize the effect of the reflections in a single-fiber CLS-PON. Then, we modulate the TL output, by means of a Mach-Zehnder intensity modulator, with an inverse RZ (IRZ) format in order to obtain a bidirectional full-duplex operation, as proposed in [21]. The TL output lightwave passes through a polarization scrambler, driven by a $6 \mathrm{kHz}$ random signal, followed by a variable optical attenuator (VOA-1), which controls the seeding light power level at the fiber input. The polariza- 


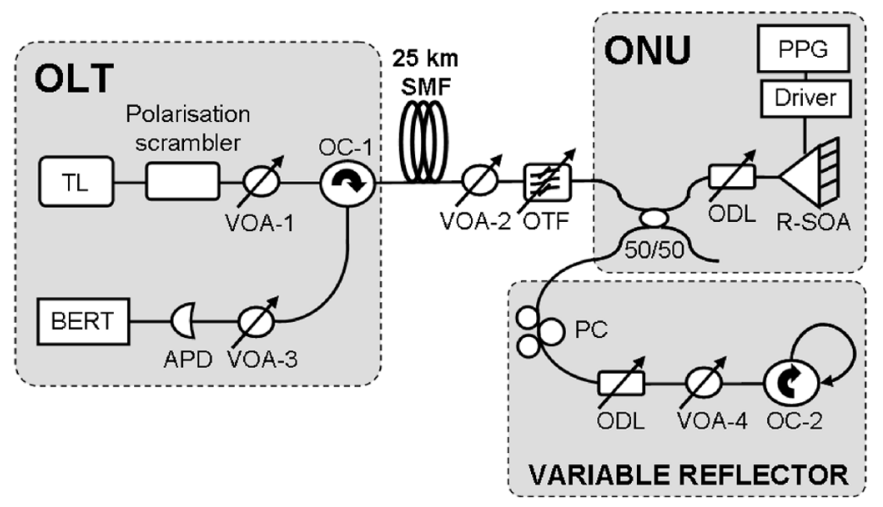

Fig. 3. Experimental setup. TL: tunable laser, VOA: variable optical attenuator, OC: optical circulator, OTF: optical tunable filter, ODL: optical delay line, R-SOA: refelective-SOA, PPG: pulse pattern generator, PC: polarization controller, APD: avalanche photodiode, and BERT: BER tester.

tion scrambler is used to average any effect due to the polarization dependency of the R-SOA. After an optical circulator (OC-1), the downstream signal is launched into a $25 \mathrm{~km}$-long single-mode fiber (SMF, $6 \mathrm{~dB}$ loss). At the output of the fiber, the seeding light passes through VOA-2, a tunable optical filter (OTF, Gaussian shape, $0.8 \mathrm{~nm} 3 \mathrm{~dB}$ bandwidth, and $4 \mathrm{~dB}$ insertion loss), a $3 \mathrm{~dB}$ coupler and a variable optical delay line (ODL), then reaches the R-SOA. In the full-duplex case, the ODL in front of the R-SOA allows for the synchronization of the downstream and the upstream signals, by interleaving the RZ upstream transmission by half-bit in respect to the incoming IRZ pattern, so that the RZ modulation is always performed over a CW slot [21]. The R-SOA is a commercial device providing 26 $\mathrm{dB}$ small signal gain, $6 \mathrm{dBm}$ output saturated power at $75 \mathrm{~mA}$ bias current and $1 \mathrm{~dB}$ polarization dependent gain working at $20^{\circ} \mathrm{C}$. We use it driven either by an NRZ or by an RZ $1.25 \mathrm{~Gb} / \mathrm{s}$ PRBS sequence (in both cases a $2^{11}-1$ bit-long pattern). The R-SOA driving voltage is chosen quite high $\left(7 V_{p-p}\right)$; this partly compensates for the limited E/O bandwidth (around $1.5 \mathrm{GHz}$ ) of the R-SOA, which is particularly useful for the RZ signal. At the same time, the high driving voltage increases the gain excursion, thus increasing the chirp significantly.

The light remodulated by the R-SOA is coupled back to the fiber and can also be sent to a variable reflector module, which emulates a source of concentrated reflection extremely close to the ONU. We note that we emulate a reflection point very close to the ONU in order to maximize the impact of the concentrated reflection on the system [8]. The variable reflector is composed by a polarization controller (PC), a variable ODL, VOA-4, and a mirror, realized by means of an optical circulator (OC-2) in a closed-loop configuration. During the experiment, by means of the ODL and the PC, we adjust the delay and the polarization state of the reflected signal in order to maximize the impact of concentrated reflection.

Finally, the modulated signal sent upstream over the SMF reaches the OLT, where it is extracted by means of OC-1, attenuated by VOA-3, and received. Our receiver is composed of an avalanche photodiode (APD, $-35 \mathrm{dBm}$ sensitivity), followed by low-pass electrical Bessel filter (LPF, $933 \mathrm{MHz}$ bandwidth). We note that in all the measurements reported in the following, the receiver threshold is adapted to the optimal position during the measurements. This usually gives better signal-to-crosstalk ratio (SCR) tolerance for both modulation formats compared to the case of a simple receiver having fixed threshold [25].

\section{EXPERIMENTAL RESULTS}

\section{A. Resilience Against the Distributed Reflections}

In order to evaluate only the impairments due to the crosstalk induced by the RBS light, we first disconnect the variable reflector module, so that only the distributed reflection from the fiber are taken into account.

We start considering a $\mathrm{CW}$ seeding light at $1535 \mathrm{~nm}$ generated from the TL. By means of VOA-1, we set the power launched into the fiber at $0 \mathrm{dBm}$, which corresponds to about $-34 \mathrm{dBm}$ back-scattered light from the fiber. The light backscattered from the SMF and the upstream signal modulated by the R-SOA travel along the same fiber in the same direction, therefore they interfere with each other at the OLT. In order to characterize the impairments due to the RBS, we define the $\mathrm{SCR}$ as the ratio between the upstream received power and the fiber back-scattered power $(-34 \mathrm{dBm})$. By means of VOA-2, we can then change the power at the input of the R-SOA. This changes the gain of the R-SOA and, in turn, the power at the input of the upstream receiver, hence the SCR value. In our case, since the fiber back-scattered light is around $-34 \mathrm{dBm}$, when the R-SOA input power is between -16 and $-24 \mathrm{dBm}$, the SCR at the OLT varies between 24 and $10 \mathrm{~dB}$. We adopt those R-SOA input power levels because they correspond respectively to the maximum power that can be delivered to the ONU (for 0 $\mathrm{dBm}$ launched power from the OLT) and the minimum power required to obtain a sensitivity degradation of about $3 \mathrm{~dB}$ for upstream RZ modulation format.

We note that, when the variable reflector is disconnected, the dominating impairment is due to the distributed reflections of the downstream CW seed toward the OLT (type-I reflections), since the effect of the back-reflections of the upstream signal back into the ONU (type-II reflections) is negligible. Indeed, considering the losses due to VOA-2, the OTF, the $3 \mathrm{~dB}$ coupler, and the ODL, the amount of back-reflected power from the fiber toward the ONU is estimated around $-50 \mathrm{dBm}$. Hence, the SCR at the ONU is always higher than $30 \mathrm{~dB}$. In this operating condition, we can consider the SCR degradation at the ONU negligible compared to the SCR degradation that occurs at the OLT, where the SCR is always lower than $24 \mathrm{~dB}$. In this configuration, we compare the performance of the RZ modulation format to the NRZ in terms of eye aperture and sensitivity degradation.

In Fig. 4, we report the eye diagrams of the received upstream for the two modulation formats and for three different SCR values $(22,16$, and $10 \mathrm{~dB})$. As we can see, the RZ signal always shows a good quality and has a clear eye opening even at $10 \mathrm{~dB}$ SCR level. On the other hand, the NRZ modulated signal is severely degraded even at much higher SCR values (around 16 $\mathrm{dB})$. As we see in the figure, the RZ eye diagrams show double slopes, which is mainly due to the limited electro-optic bandwidth of the R-SOA $(1.5 \mathrm{GHz})$.

This difference in the performance is clearly shown in Fig. 5, where we report the measured upstream receiver sensitivity, at 


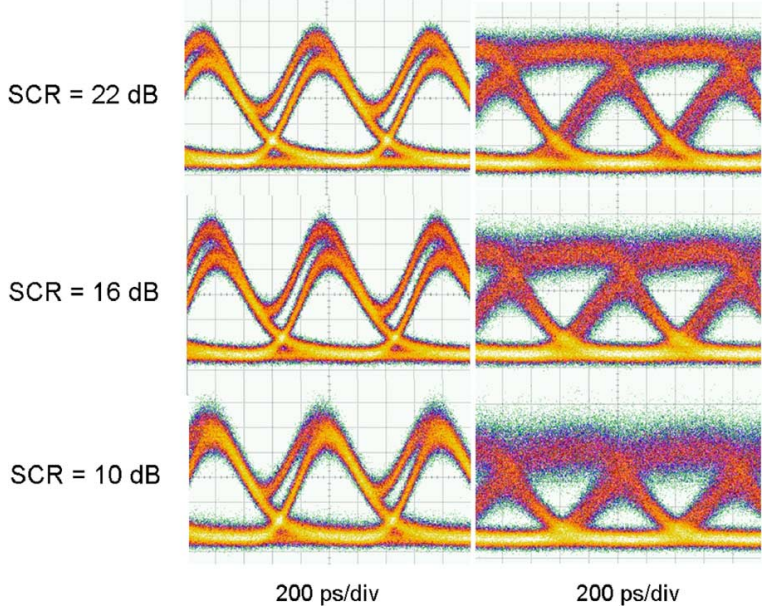

Fig. 4. Eye diagrams of the RZ (1st column) and the NRZ (2nd column) upstream signals at $1.25 \mathrm{~Gb} / \mathrm{s}$, in presence of distributed reflections. In each case, the eye diagrams are taken for 22,16 , and $10 \mathrm{~dB}$ SCR values.

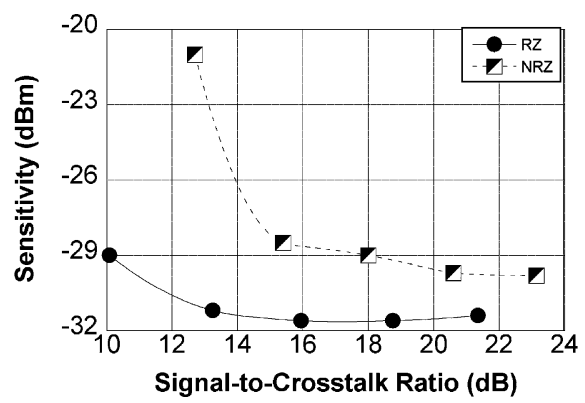

Fig. 5. Resilience against the distributed reflection for the RZ (circles) and the NRZ (squares) upstream transmission at $1.25 \mathrm{~Gb} / \mathrm{s}$. The curves report the sensitivity (at $\mathrm{BER}=10^{-9}$ ) versus the SCR value.

bit error rate $(B E R)=10^{-9}$, as a function of the SCR for the RZ (circles) and the NRZ signals (squares). As we can see, the NRZ signal requires a SCR level higher than around $16 \mathrm{~dB}$, in order to maintain a sensitivity penalty lower than $2 \mathrm{~dB}$. Above that SCR value, the penalty increases abruptly. On the other hand, when using the RZ format we can increase dramatically the resilience to the crosstalk: a BER of $10^{-9}$ is observed up to $10 \mathrm{~dB}$ SCR level, with a sensitivity penalty lower than $3 \mathrm{~dB}$.

In this configuration, we measure also the dependence of the receiver sensitivity as a function of the wavelength of the $\mathrm{CW}$ seeding light for the two modulation formats. In this analysis, we fix the path loss at $22 \mathrm{~dB}$, so that the R-SOA input power is always $-22 \mathrm{dBm}$. Comparing the two modulation formats, we must recall that the R-SOA output power is different in the two cases: when we use the NRZ modulation, the output power is more than $1 \mathrm{~dB}$ higher in respect to the RZ. This is due to the different duty-cycle of the RZ pulse in respect to the NRZ, which results in an increased mean power for the NRZ format by more than $1 \mathrm{~dB}$. In this condition, the SCR value at the receiver for the NRZ format is in the range between 15 and $14 \mathrm{~dB}$ on the whole measurement bandwidth, whereas the SCR for the RZ format is only between 14 and $13 \mathrm{~dB}$.

Even though the RZ format has a reduced SCR, the sensitivity value is lower than the NRZ at every wavelength, as shown in Fig. 6, where we report the sensitivity (at BER $=10^{-9}$ ) as

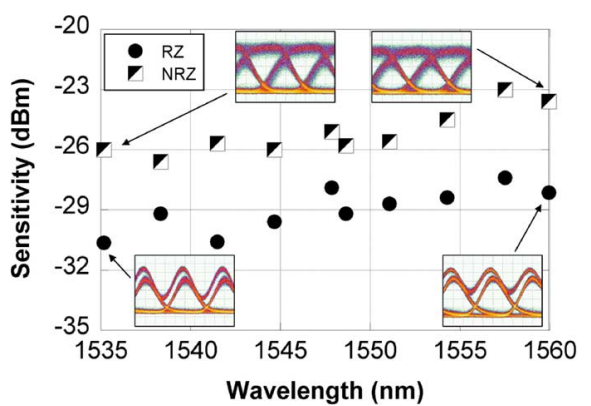

Fig. 6. Sensitivity (at BER $=10^{-9}$ ) as a function of wavelength for the RZ (circles) and the NRZ (squares) upstream transmission at 1.25 Gbits/s. Insets: eye diagrams of the RZ and the NRZ signals taken at $1530 \mathrm{~nm}$ and $1560 \mathrm{~nm}$. The measurements were taken at fixed power $(-22 \mathrm{dBm})$ at the R-SOA input.

a function of the wavelength when the upstream is an highly chirped RZ signal (circles) or a NRZ signal (squares). In the same graph, we can also see that, for both formats, the sensitivity degrades when the wavelength increases. This is due to two reasons: first, the gain profile of the R-SOA has its maximum around $1535 \mathrm{~nm}$, which results into a lower output power at longer wavelengths which reduces the SCR at the receiver. Second, the R-SOA electro-optical bandwidth slightly decreases at longer wavelengths; this distorts the shape of the R-SOA-modulated signal. We can see this effect in the insets in Fig. 6 where the eye diagrams for the RZ and the NRZ signals are reportedly taken at $1530 \mathrm{~nm}$ and $1560 \mathrm{~nm}$.

\section{B. Resilience Against the Concentrated Reflections}

The impact of the concentrated reflections is analyzed using the experimental setup reported in Fig. 3. In order to evaluate only the impact of concentrated reflections on the system, we remove the $25-\mathrm{km}$ fiber spool and we connect the variable reflector module. The variable reflector emulates a concentrated reflection extremely close to the ONU transmitter (few meters), which is known to be far more detrimental than a distributed Rayleigh reflector [8]. In order to account for the concentrated reflections, we define a network return loss (NRL) parameter as the ratio between the reflected power from the variable reflector that re-enters the R-SOA and the R-SOA output power. By acting on VOA-4, we can set the NRL values between -55 and $-20 \mathrm{~dB}$. Both the PC and the variable ODL in the reflector module are set to maximize the impact of those reflections by maximizing the BER degradation for both the modulation formats. The resilience against the concentrated reflections is reported in Fig. 7, where we compare the sensitivity at $\mathrm{BER}=10^{-9}$ for the two modulation formats. We perform the measurements using two different input power levels at the R-SOA: -16 and $-24 \mathrm{dBm}$. In both cases, the RZ format (circles) outperforms the NRZ (squares), which cannot tolerate NRL level greater than $-30 \mathrm{~dB}$. At this value of NRL where the NRZ modulation format cannot work, the RZ format still shows a limited penalty in terms of sensitivity (about $2 \mathrm{~dB}$ ) for both input power levels. In the insets in Fig. 7, we report the eye diagrams for the RZ and the NRZ modulation format recorded at highest tolerable NRL values. As can be seen in the insets, the RZ format can tolerate higher values of NRL, with respect to the NRZ, preserving a good eye aperture for both values of the input power. However, we note that when 




Fig. 7. Resilience against the concentrated reflection for the RZ (circles) and the NRZ (squares) upstream transmission at $1.25 \mathrm{~Gb} / \mathrm{s}$. The curves report the sensitivity (at BER $=10^{-9}$ ) versus the network return loss parameter for two different values of R-SOA input power: -16 and $-24 \mathrm{dBm}$. Insets: uplink signals at $1.25 \mathrm{~Gb} / \mathrm{s}$, in presence of concentrated reflections.

the R-SOA is highly saturated ( $-16 \mathrm{dBm}$ input power), the tolerance to the concentrated reflections increases for both modulation formats. Indeed, at higher input power the R-SOA gain is more saturated and, consequently, the reflected light experiences reduced gain into the R-SOA. This increased resilience to the concentrated reflections allows the use of components having worse return loss, which, in principle, make the system more cost effective.

\section{Full-Duplex Symmetrical PON}

In order to asses the effectiveness of the proposed technique, we consider the effects of the RZ modulation in reducing the transmission impairments due to the reflections in a PON architecture where both the upstream and the downstream are modulated signals. We use the PON architecture presented in [21 ]. The full-duplex symmetrical operation is realized providing a $50 \%$ IRZ format downstream signal and a RZ upstream signal.

The synchronization between the downstream and the upstream signal is obtained by adjusting the ODL in front of the $\mathrm{R}-\mathrm{SOA}$ at the ONU. In a practical system, the synchronization would be achieved electronically at the ONU. We demonstrate that the synchronization between the downstream and the upstream signal is not a critical issue by introducing a delay on the remodulation position by means of the ODL. In Fig. 8 , we report the sensitivity (at BER $=10^{-9}$ ) penalty as a function of the introduced delay in respect to the optimal synchronization $(0 \mathrm{ps})$. As it can be seen from the figure, only $2 \mathrm{~dB}$ of penalty in terms of sensitivity is obtained for a synchronization mismatch of more than $\pm 200 \mathrm{ps}$, which corresponds to more than $50 \%$ of the bit period $(800 \mathrm{ps})$.

In the full-duplex symmetrical PON, the resilience against the distributed reflections is evaluated using the setup reported in Fig. 3, removing the variable reflector module. In these measurements, we choose the TL wavelength close to the R-SOA gain peak $(1535 \mathrm{~nm})$. During the full-duplex operation, the launch power from the OLT into the fiber is $0 \mathrm{dBm}$, which corresponds to about $-34 \mathrm{dBm}$ of back-scattered power from the fiber. Basically, in this PON architecture we have two

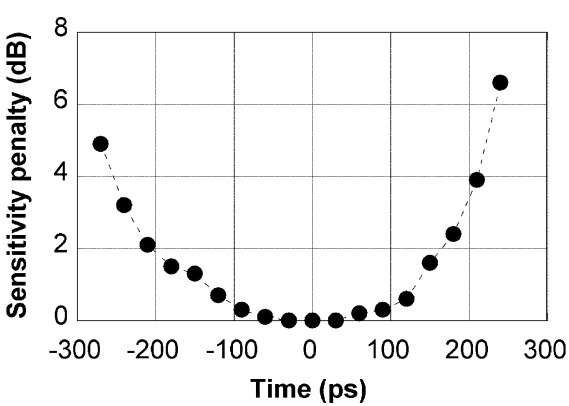

Fig. 8. Synchronization error tolerance in the full-duplex operation. The curve reports the sensitivity penalty as a function of the introduced delay with respect to the optimal synchronization $(0 \mathrm{ps})$.

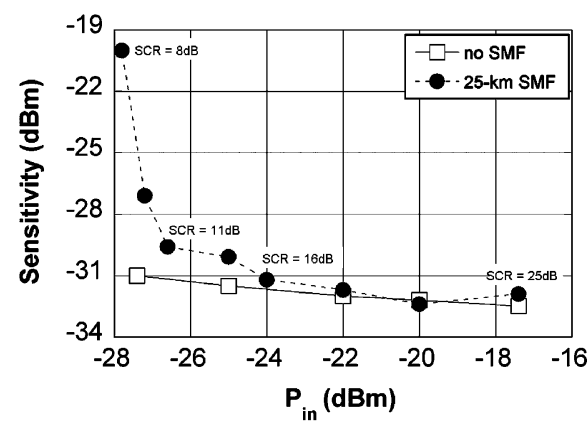

Fig. 9. Resilience to the distributed reflections of the RZ upstream signal in the full-duplex operation. The curves report the sensitivity (at BER $=10^{-9}$ ) versus the R-SOA input power when the OLT is directly connected to the ONU (squares) and when $25 \mathrm{~km}$ of SMF spool is inserted between the two terminals (circles)

sources of interference: the RBS of downstream signal directed toward the OLT (type-I reflections) and the RBS of upstream signal toward the ONU (type-II reflections).

First, we analyze the performance of the chirped RZ upstream signal in presence of type-I reflections in respect to the case with no reflections. For this analysis, we compare the sensitivity of the upstream signal when the ONU is directly connected to the OLT (no reflections) and when a $25 \mathrm{~km} \mathrm{SMF} \mathrm{spool} \mathrm{is} \mathrm{between}$ the two terminals (type-I reflections). In Fig. 9, we report the results in terms of sensitivity (at BER $=10^{-9}$ ) as a function of the R-SOA input power in the two cases: with no reflections (squares) and in presence of type-I reflections (circles). As it can be seen comparing the two curves in Fig. 9, only $2 \mathrm{~dB}$ of sensitivity penalty is obtained when the R-SOA input power is reduced down to $-27 \mathrm{dBm}$, which corresponds to about $11 \mathrm{~dB}$ of SCR and no penalty is observed for a wide range of R-SOA input power: from -17 to $-24 \mathrm{dBm}$ (SCR between 25 and 16 $\mathrm{dB})$. We note that, the receiver threshold is again adapted to the optimal position during the measurements.

In the full-duplex operation, we characterize the performance of the system by measuring also the receiver sensitivity in presence of distributed reflections as a function of the wavelength, for both the downstream and the upstream signals. This characterization is performed changing the TL operating wavelength. During this analysis, we adjust the path attenuation by means of VOA-2 in order to keep the SCR value at the OLT fixed at $12 \mathrm{~dB}$ at every wavelength. In this operating condition, considering the loss induced by the VOA-2, the OTF, the $3-\mathrm{dB}$ coupler, and the 


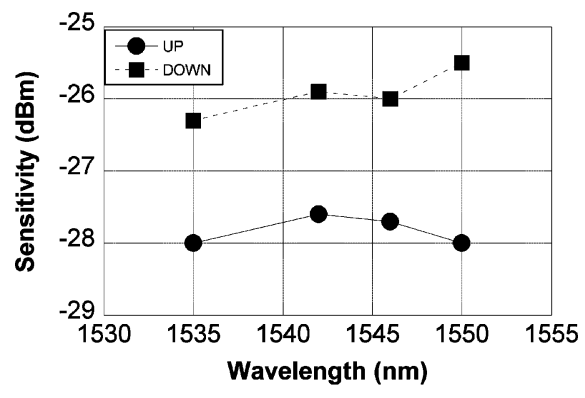

Fig. 10. Sensitivity in the full-duplex PON as a function of wavelength. The curves report the data taken for the upstream RZ (circles) and for the downstream IRZ (squares) signals. The measurements were taken at fixed signal-to-crosstalk ratio $(12 \mathrm{~dB})$.

ODL, the power of the back-reflected upstream that interfere at the ONU with the downstream signal is lower than $-60 \mathrm{dBm}$. So that, the SCR value at the ONU is always higher than $40 \mathrm{~dB}$, thus we can neglect the interference due to the type-II reflections. At these SCR values, we measure the sensitivity values as a function of the wavelength, for both the upstream and the downstream signals. Those measurements are performed using the receiver described in Section III , optimizing the receiver threshold during the measurements. In Fig. 10, we report the sensitivity (at BER $=10^{-9}$ ) of the downstream and the upstream signals as a function of wavelength. As we can see from the figure, the sensitivity for the IRZ downstream (squares) is more than $1 \mathrm{~dB}$ higher than the RZ upstream signal (circles) at every wavelength. This lower sensitivity is typical of any IRZ signal. The wavelength tendency of the upstream signal reported in Fig. 10 shows a slightly reduced sensitivity for longer wavelengths (about $1 \mathrm{~dB}$ ). Nevertheless, from the figure we can observe that no substantial degradation of the sensitivity occurs when the operating wavelength is varied from 1535 to $1550 \mathrm{~nm}$ for both the IRZ downstream and the RZ upstream signals, this provides for a wide operating bandwidth.

\section{CONCLUSION}

We investigate how the RZ modulation can alleviate the transmission impairments due to the reflections in R-SOA-based bidirectional PONs. We experimentally assess the resilience to the distributed and to the concentrated reflections when the $\mathrm{R}-\mathrm{SOA}$ is RZ or NRZ modulated. When the distributed reflections are taken into account, the chirped RZ upstream signal performs better than the NRZ for a wide range of SCR values. This is more effective when the wavelength of the seeding light is chosen close to the maximum of the R-SOA gain profile. Similarly, the resilience to the concentrated reflections also improves for the chirped RZ signal, which tolerates return loss values worse than $-25 \mathrm{~dB}$. The higher reflection resilience of the chirped RZ upstream signal, is then confirmed in a full-duplex PON, i.e., having also the downstream signal modulated. We found that, in a full-duplex symmetrical PON, the upstream transmission of a chirped RZ signal can improve the resilience against the distributed reflection down to SCR value of $11 \mathrm{~dB}$ with a reduced sensitivity penalty $(2 \mathrm{~dB})$.

These results indicate that the use of highly chirped RZ signals in R-SOA-based PONs can be more robust against the in-band coherent crosstalk generated by both the distributed and the concentrated reflections. Moreover this techniques is demonstrated to be effective for a wide range of wavelengths, therefore, in principle, the proposed solution could be useful also in WDM-PONs.

\section{REFERENCES}

[1] L. Kazovsky, W. Shaw, D. Gutierrez, N. Cheng, and S. Wong, "Nextgeneration optical access networks," J. Lightw. Technol., vol. 25, no. 11, pp. 3428-3442, Nov. 2007.

[2] J. Prat, Next-Generation FTTH Passive Optical Networks: Research Towards Unlimited Bandwidth Access. New York: Springer-Verlag, 2008.

[3] C. Chan, L. Chem, and C. Lin, "WDM PON for next-generation optical broadband access networks," in Proc. Opto-Electron. Commun. Conf. (OECC), 2006, pp. 1-3.

[4] F. Payoux, P. Chanclou, and N. Genay, "WDM-PON with colorless onus," in Proc. Opt. Fiber Commun. Conf., Mar. 2007, pp. 1-3.

[5] R. Staubli and P. Gysel, "Crosstalk penalities due to coherent rayleigh noise in bidirectional optical communication systems , J. Lightw. Technol., vol. 9, no. 3, pp. 375-380, Mar. 1991.

[6] M. Fujiwara, J. Kani, H. Suzuki, and K. Iwatsuki, "Impact of backreflection on upstream transmission in WDM single-fiber loopback access networks," J. Lightw. Technol., vol. 24, no. 2, pp. 740-746, Feb. 2006.

[7] C. Arellano, K.-D. Langer, and J. Prat, "Reflections and multiple Rayleigh backscattering in WDM single-fiber loopback access networks ," J. Lightw. Technol., vol. 27, no. 1, pp. 12-18, Jan. 1, 2009.

[8] Y. Lee, K. Cho, A. Murakami, A. Agata, Y. Takushima, and Y. Chung, "Reflection tolerance of RSOA-based WDM PON," in Proc. Opt. Fiber Commun. Conf. (OFC)/Natl. Fiber Optic Eng. Conf. (NFOEC'08), pp. $1-3$.

[9] K. Y. Cho, Y. J. Lee, H. Y. Choi, A. Murakami, A. Agata, Y. Takushima, and Y. C. Chung, "Effects of reflection in RSOA-based WDM PON utilizing remodulation technique," J. Lightw. Technol., vol. 27, no. 10, pp. 1286-1295, May 2009.

[10] M. Sumida, T. Kubo, and T. Imai, "Limitations imposed by rayleigh backscattering in closely interleaved, bidirectional WDM transmission systems," IEEE Photon. Technol. Lett., vol. 15, no. 1, pp. 150-152, Jan. 2003.

[11] H. Hu and H. Anis, "Degradation of bidirectional single fiber transmission in WDM-PON due to beat noise," J. Lightw. Technol., vol. 26, no. 8, pp. 870-881, Apr. 2008.

[12] P. Urban, A. Koonen, G. Khoe, and H. de Waardt, "Interferometric crosstalk reduction in an RSOA-based WDM passive optical network," J. Lightw. Technol., vol. 27, no. 22, pp. 4943-4953, Nov. 2009.

[13] S. Das and E. Harstead, "Beat interference penalty in optical duplex transmission," J. Lightw. Technol., vol. 20, no. 2, pp. 213-217, Feb. 2002.

[14] Y. Shen, K. Lu, and W. Gu, "Coherent and incoherent crosstalk in WDM optical networks," J. Lightw. Technol., vol. 17, no. 5, pp. 759 -764, May 1999.

[15] C. Marki, F. Marki, and S. Esener, "Reduction of interferometric optical crosstalk penalty via dc blocking," Electron. Lett., vol. 43, no. 11, pp. 644-646, May 2007.

[16] A. Chiuchiarelli, M. Presi, R. Proietti, G. Contestabile, P. Choudhury, L. Giorgi, and E. Ciaramella, "Enhancing resilience to rayleigh crosstalk by means of line coding and electrical filtering " IEEE Photon. Technol. Lett., vol. 22, no. 2, pp. 85-87, Jan. 15, 2010.

[17] K. Cho, A. Murakami, Y. Lee, A. Agata, Y. Takushima, and Y. Chung, "Demonstration of RSOA-based WDM PON operating at symmetric rate of $1.25 \mathrm{~Gb} / \mathrm{s}$ with high reflection tolerance," in Proc. Opt. Fiber Commun./Nat. Fiber Opt. Eng. Conf. (OFC/NFOEC'08), Feb., pp. 1-3.

[18] P. Urban, A. Koonen, G. Khoe, and H. de Waardt, "Coherent crosstalksuppression in WDM access networks employing reflective semiconductor optical amplifier," in Proc. IEEE Eur. Conf. Opt. Commun. (ECOC'07), pp. 91-92

[19] J. Lee, D. Lee, Y. Won, S. Park, and S. Han, "Reduction of Rayleigh back-scattering noise using RF tone in RSOA based bidirectional optical link," presented at the Opt. Fiber Commun. Conf., San Diego, CA, 2008.

[20] L. Banchi, R. Corsini, M. Presi, F. Cavaliere, and E. Ciaramella, "Enhanced reflection tolerance in WDM-PON by chirped RZ modulation," Electron. Lett., vol. 46, no. 14, pp. 1009 -1011, Jul. 2010.

[21] M. Presi, R. Proietti, K. Prince, G. Contestabile, and E. Ciaramella, "A $80 \mathrm{~km}$ reach fully passive WDM-PON based on reflective onus," Opt. Exp., vol. 16, no. 23, pp. 19043-19048, 2008.

[22] C. Henry, "Theory of the linewidth of semiconductor lasers," IEEE $J$. Quantum Electron., vol. 18, no. 2, pp. 259-264, Feb. 1982. 
[23] L. Occhi, L. Schares, and G. Guekos, "Phase modeling based on the alpha;-factor in bulk semiconductor optical amplifiers," IEEE J. Sel. Top. Quantum Electron., vol. 9, no. 3, pp. 788-797, May-Jun. 2003.

[24] D. Derickson, Fiber Optic Test and Measurement. Upper Saddle River, NJ: Prentice-Hall, 1998.

[25] E. Goldstein, L. Eskildsen, and A. Elrefaie, "Performance implications of component crosstalk in transparent lightwave networks," IEEE Photon. Technol. Lett., vol. 6, no. 5, pp. 657-660, May 1994.

Luca Banchi was born in Livorno, Italy, in 1981. He received the Laurea (M.Sc.) degree in telecommunication engineering from the University of Pisa, Pisa, Italy, in June 2005 . He is currently working toward the Ph.D. degree at Scuola Superiore SantAnna, Pisa, Italy.

Between September 2005 and December 2007, he was with Elettra Sicrotrone Trieste working on the optical synchronization system of FERMI Free Electron Laser. His research is focused on the new technical solutions for advanced wavelength division multiplexing systems.

Raffaele Corsini received the Laurea degree in physics from the University of Milan, Milan, Italy, in 1992.

He has 17 years working experience in R\&D in the Pirelli group and one in PGT Photonics (subsidiary of CyOptics, Inc.), in the field of optical and opto-electronics components for fiber-optic telecommunication systems. In this period, he developed a deep knowledge of the technical aspects related to optical devices, technology, and fiber-optic systems. Since 2010, he has been a Research Associate at Scuola Superiore SantAnna, Pisa, Italy, where he currently works on fiber-optic and free space transmission systems.
Marco Presi received the Laurea degree in physics from the University of Rome La Sapienza, Rome, Italy, in 2001, and the Ph.D. degree in applied physics from the University of Pisa, Pisa, Italy, in 2006.

Since 2007, he has been a Research Associate at Scuola Superiore SantAnna, Pisa, Italy, where he currently works on wavelength division multiplexing optical systems. He coauthored more than 50 papers published in international peer-reviewed journals and in international conferences.

Ernesto Ciaramella (M’06) received the Laurea degree from La Sapienza University of Rome, Rome, Italy, in 1991.

$\mathrm{He}$ is working at Fondazione Ugo Bordoni (FUB) and Centro Studi e Laboratorie Telecommunicazioni (CSELT), on nonlinear optical effects, design of optical transmission systems, and network architectures. During 2001-2002, he was a Research Manager at the Consorzio Nazionale Interuniversitario per le Telecomunicazioni (CNIT) National Photonic Networks Laboratory, Pisa, Italy. Since 2002, he has been an Associate Professor with the Scuola Superiore SantAnna, University of Pisa, Pisa, Italy. His current research interest include wavelength division multiplexing systems for transport and access networks, optical processing, and free space optics systems. He has authored or coauthored approximately 140 papers and holds ten international patents. $\mathrm{He}$ has served on the programme committees for many international conferences. 